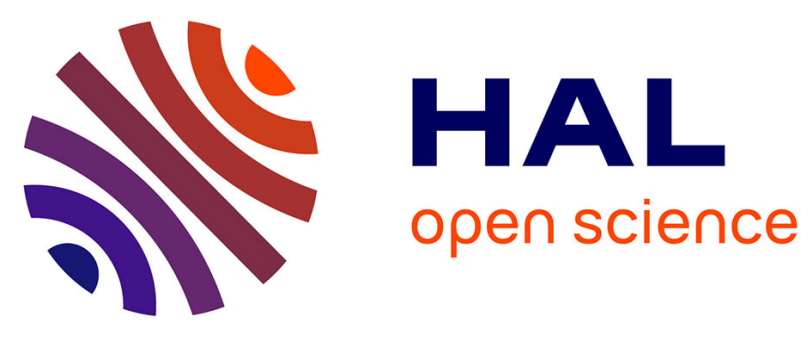

\title{
Precision of proportion estimation with binary compressed Raman spectrum
}

Philippe Réfrégier, Camille Scotté, Hilton B de Aguiar, Herve Rigneault, Frédéric Galland

\section{> To cite this version:}

Philippe Réfrégier, Camille Scotté, Hilton B de Aguiar, Herve Rigneault, Frédéric Galland. Precision of proportion estimation with binary compressed Raman spectrum. Journal of the Optical Society of America. A Optics, Image Science, and Vision, 2018, 35 (1), 10.1364/JOSAA.35.000125 . hal01715283

\section{HAL Id: hal-01715283 \\ https://hal.science/hal-01715283}

Submitted on 27 Feb 2018

HAL is a multi-disciplinary open access archive for the deposit and dissemination of scientific research documents, whether they are published or not. The documents may come from teaching and research institutions in France or abroad, or from public or private research centers.
L'archive ouverte pluridisciplinaire HAL, est destinée au dépôt et à la diffusion de documents scientifiques de niveau recherche, publiés ou non, émanant des établissements d'enseignement et de recherche français ou étrangers, des laboratoires publics ou privés. 


\title{
Precision of proportion estimation with binary compressed Raman spectrum
}

\author{
Philippe Réfrégier ${ }^{1}$, Camille Scotté ${ }^{1}$, Hilton B. de Aguiar ${ }^{1,2}$, Hervé Rigneault ${ }^{1}$, And \\ FrÉdÉRIC GALLAND ${ }^{1, *}$ \\ ${ }^{1}$ Aix Marseille Univ, CNRS, Centrale Marseille, Institut Fresnel, Marseille, France \\ ${ }^{2}$ Département de Physique, Ecole Normale Supérieure / PSL Research University, CNRS, 24 rue Lhomond, 75005 Paris, France \\ ${ }^{*}$ Corresponding author: frederic.galland@fresnel.fr
}

Compiled November 5, 2017

\begin{abstract}
The precision of proportion estimation with binary filtering of Raman spectrum mixture is analyzed when the number of binary filters is equal to the number of present species and when the measurements are corrupted with Poisson photon noise. It is shown that the Cramer-Rao bound provides a useful methodology to analyze the performance of such an approach and in particular when the binary filters are orthogonal. It is demonstrated that a simple linear mean square error estimation method is efficient (i.e. has a variance equal to the Cramer-Rao Bound). Evolutions of the Cramer-Rao bound are analyzed when the measuring times are optimized or when the considered proportion for binary filter synthesis are not optimized. Two strategies for the appropriate choice of this considered proportion are also analyzed for the binary filter synthesis. () 2017 Optical Society of America
\end{abstract}

OCIS codes: $\quad 070.4560$ - Data processing by optical means, 110.4280 - Noise in imaging systems, 110.3055 - Information theoretical analysis, 300.6450 - Spectroscopy Raman.

http://dx.doi.org/10.1364/ao.XX.XXXXXX

\section{INTRODUCTION AND BACKGROUND}

\section{A. Introduction}

Spontaneous Raman spectroscopy allows one to probe a chemically heterogeneous system with high molecular specificity. In its most general implementation, Raman inelastically scattered light is collected and spectrally dispersed on sensitive array detectors (e.g. charge-coupled device, CCD). As the Raman cross section is relatively weak, this methodology requires typically long acquisition times to overcome noise associated with detection hardware. To overcome these limits, new strategies have been recently implemented, mostly focused on spectrally multiplexed measurements that may enable faster or shot-noise limited performance [1-6]. Nevertheless, these "full spectrum" multiplexing methodologies still acquire data which might be not necessary for certain applications, such as in sorting chemical species whose Raman spectra are known.

An alternative approach based on compressive detection has been proposed recently [4,7-10] when the measured spectra is a linear combination of elementary spectrum of different species. In this methodology, proportion of known species can be estimated from few measurements (at least as many measurements as the number of species). In this framework, each measurement is performed using programmable binary spectral filters and a single channel detector for each filter. The speed may thus surpass array detectors (i.e. CCD) with lower acquisition times or noise level for chemical species quantitative analysis, hence enabling faster image acquisition using the spontaneous Raman process.

In this article, we consider the problem of retrieving unknown proportions from a mixture of known species from compressed spectra that are obtained with measuring the intensity through optical binary filters. It is assumed that the mixed spectrum is a linear combination of elementary known spectra with unknown coefficients $z_{i}$ (see for example [4, 7-11] and references herein). The goal is thus to investigate precisely the performance of the compressed Raman method in comparison to full Raman techniques (i.e. raster scanning the grating of a spectrometer or using a CCD detector) when measuring such kinds of mixed spectra. Issues of practical implementation of optical filtering of Raman spectra have been discussed in particular in $[1,2,4-7,9]$ and the purpose of this paper is to investigate theoretical information properties of the compressed Raman technique, without analyzing technological limitations, but with introducing fundamental physical limits such as photon noise. This approach allows one to analyse the information properties of both the full and the compressed Raman methods, when only the fundamental photon noise is taken into account. 
This study is thus independent of particular implementation limits such as hardware-related noise sources. However, generalizing the results in the presence of additive electronic noise and of background noise is easy as discussed in an appendix. For that problem, we analyze the ultimate precision described by the Cramer-Rao lower bound (CRB). Analyzing the CRB has the advantage of being independent of the choice of the unbiased estimation algorithm that processes the number of photons measured with each binary filter. Although the general expression of the CRB can be applied when the number of filters is larger than the number of known species with unknown proportion, two particular cases are precisely investigated. Indeed, a particular attention is provided to situations for which the number of filters is equal to the number of known species. It is demonstrated that, in that case, a simple linear mean square error estimation method [7-10] is efficient (i.e. has a variance equal to the Cramer-Rao Bound). Estimation performance is also analyzed with Orthogonal Binary Filters (OBF) that constitute a subset of Binary Filters (BF) [7-10] and that are defined so that for each frequency at most one filter is equal to 1 . Although practically difficult, the OBF method allows one to implement the filters in parallel when the measuring times for each binary filter are equal. In that case, it is possible to precisely compare the obtained precision with the standard Full Spectral Raman (FSR) method. The usefulness of the CRB for proportion estimation based on full Raman spectra has been illustrated in [11] and its comparison with the CRB obtained with OBF allows one to quantify the efficiency of this last technique. Bounds on the Cramer-Rao bound with BF techniques are then analyzed as well as strategies for the choice of the considered proportions for the binary filters synthesis. These results show that a simple methodology can be implemented to optimize binary filters for each particular application.

In order to analyze the information performance of the BF and OBF approaches and to avoid bias due to any imperfect experimental condition, the study is performed with numerical simulations. The numerical results are obtained with the considered spectrum examples but the proposed analysis provides a general methodology for other application examples or experimental conditions.

In subsection 1.B the compressed Raman technique and the noise model is summarized. Then, in Section 2, the CramerRao bound (CRB) is determined for compressed Raman technique and the optimization method for binary filter synthesis is presented as well as numerical simulation results for different types of spectra. Then, the efficiency of a simple linear method analogous to the one introduced in [7-10] is demonstrated when the number of binary filters is equal to the number of elementary species. This assumption of equality of the number of binary filters and of the number of elementary species is thus considered in the following of the paper. The advantage of optimizing measuring times for each binary filter is then investigated and bounds on the CRB are provided. Section 3 is devoted to the comparison of the compressed Raman technique with the full Raman approach. Since the binary filters are generated for particular proportion values, the robustness of the technique to the choice of these values is also discussed using the CRB of both the compressed and full Raman techniques. The influence of the particular proportions used for the binary filters design is discussed in section 4 . Conclusion and perspectives are provided in section 5 and mathematical proofs and extensions are developed in the appendices.

\section{B. Background}

Let $N_{\tau}(v)$ denote the number of photons measured with a full Raman process in the frequency bandwidth $\left[v-\delta_{v} / 2, v+\delta_{v} / 2\right]$ with acquisition time $\tau$. The statistical average value $\left\langle N_{\tau}(v)\right\rangle$ of this number of photons can be written $\left\langle N_{\tau}(v)\right\rangle=\tau S(v)$ where $S(v)$ is proportional to the integral of the Raman spectrum for frequencies between $v-\delta_{v} / 2$ and $v+\delta_{v} / 2$. The measurements are performed for $K$ sampled frequencies $v_{k}$ with $k=1, . ., K$ so that $v_{k+1}-v_{k} \geq \delta_{v}$. The average total measured number of photons is thus $\mathcal{N}_{\tau}=\sum_{k=1}^{K}\left\langle N_{\tau}\left(v_{k}\right)\right\rangle$. The spectrum $S(v)$ is assumed to be a linear combination of $Q$ known spectra $S_{i}(v)$ so that

$$
S(v)=\sum_{i=1}^{Q} z_{i} S_{i}(v)
$$

where the coefficients $z_{i}$ can be considered as proportion coefficients that are unknown. It will be also assumed that $S_{i}(v) \geq 0$. This is a natural assumption if $S_{i}(v)$ is a spectral density but it would not have been the case if only the linear combination $S(v)=\sum_{i=1}^{Q} z_{i} S_{i}(v)$ is constrained to represent spectral densities. The $z_{i}$ coefficients are proportional to the proportion of photons emitted by species number $i$. This equation is thus based on the assumption that the mixing of species does not modify the emission spectra $S_{i}(v)$ in the range of concentration variation that are considered for the estimation. Let $\mathcal{Z}$ denote the variation domain of the $z_{i}$ and $z_{i}^{\text {sup }}$ denote the maximal values of $z_{i}$ in $\mathcal{Z}$. Let us introduce $z_{i}=z_{i}^{\prime} z_{i}^{\text {sup }}$ then clearly $z_{i}^{\prime} \leq 1$ and Eq. (1) can thus be written

$$
S(v)=\sum_{i=1}^{Q} z_{i}^{\prime} z_{i}^{\text {sup }} S_{i}(v)=\sum_{i=1}^{Q} z_{i}^{\prime} S_{i}^{\prime}(v)
$$

with the constraint that $0 \leq z_{i}^{\prime} \leq 1$. Thus, assuming that proper normalization has been performed, it will be considered below that $0 \leq z_{i} \leq 1 \quad \forall i=1, . ., Q$. Furthermore, the constraint $\sum_{i=1}^{Q} z_{i}=1$ will also sometimes be considered. The set of $z$ (with $z=\left(z_{1}, z_{2}, \ldots, z_{Q}\right)^{T}$ where ${ }^{T}$ denote the transpose operation) that satisfy $0 \leq z_{i} \leq 1 \forall i=1, . ., Q$ and $\sum_{i=1}^{Q} z_{i}=1$ will be noted $\mathcal{T}$ in the following. This condition will be used below for some numerical simulations in section 3.D and for some theoretical and numerical results in section 4 . It is also interesting to notice that, with this model, one of the elementary spectra can be a background spectrum.

It has been shown [7-10] that linear function of the proportion $z_{i}$, can be estimated when the intensity is not measured for each $v_{k}$ but through $M$ binary filters $F_{m}(v)(m=1, \ldots, M)$ with $M \geq Q$. Binary filters, defined so that $F_{m}(v)=0$ or 1 , are used for optical implementation simplicity reasons and their optimality has been discussed in $[7,8]$.

The measured number of photons $n_{m}$ through filter number $m$ is a random variable and, with standard Raman emission, its probability law can be assumed to be a Poisson distribution when the measurements are limited by photon noise. In that case, the probability to observe $n_{m}$ photons is:

$$
P_{m}\left(n_{m}\right)=e^{-\mu_{m}} \frac{\mu_{m}^{n_{m}}}{n_{m} !}
$$

where $a !=1 \times 2 \times \ldots \times a$ and $\mu_{m}=\left\langle n_{m}\right\rangle$, and thus:

$$
\mu_{m}=\tau_{m} \sum_{k=1}^{K} F_{m}\left(v_{k}\right) S\left(v_{k}\right)
$$


where $\tau_{m}$ is the measuring time for filter $\mathbf{F}_{m}=$ $\left(F_{m}\left(v_{1}\right), F_{m}\left(v_{2}\right), \ldots, F_{m}\left(v_{K}\right)\right)^{T}$. Using Eq. (1) into Eq. (4) leads to $[4,7]$ :

$$
\mu_{m}=\sum_{i=1}^{Q} G_{m i} z_{i}
$$

with $G_{m i}=\tau_{m} g_{m i}$ and $g_{m i}=\sum_{k=1}^{K} F_{m}\left(v_{k}\right) S_{i}\left(v_{k}\right)$. Introducing the matrix $\mathbf{G}$ so that $[\mathbf{G}]_{m i}=G_{m i}$, Eq. (5) can be written

$$
\mu=\mathrm{G} z
$$

where $\boldsymbol{\mu}=\left(\mu_{1}, \mu_{2}, \ldots, \mu_{M}\right)^{T}$. Eq. (6) suggests that $z$ can be estimated from $\mu$ if $\mathbf{G}^{T} \mathbf{G}$ is non singular [4, 7].

\section{CRAMER-RAO BOUND}

\section{A. Cramer-Rao bound with binary filters}

A standard statistical approach consists in looking at unbiased estimations with minimal variance [12]. If $\widehat{z}=\left(\widehat{z}_{1}, \widehat{z}_{2}, \ldots, \widehat{z}_{Q}\right)^{T}$ is an estimation of $z$, then this estimator is unbiased if $\langle\widehat{z}\rangle=z$. The precision of an unbiased estimator can be characterized by its covariance matrix $\boldsymbol{\Gamma}=\left\langle\delta \widehat{\boldsymbol{z}} \delta \widehat{\boldsymbol{z}}^{T}\right\rangle$ with $\delta \widehat{\boldsymbol{z}}=\widehat{\boldsymbol{z}}-\langle\boldsymbol{z}\rangle$. The $M$ filters $F_{m}(v)$ can thus be determined in order to optimize a figure of merit deduced from $\Gamma$ such as the mean square error of $\widehat{z}$ obtained with a linear function of the measures $n_{m}$ [7-9]. Complementary information can be obtained with analyzing the CRB. Such an approach has been developed in [11] for standard Raman spectroscopy perturbed with Poisson noise. It is shown in the following that it can be helpful for binary compressed Raman spectrum. Indeed, for any unbiased estimator:

$$
\left\langle\delta \widehat{z}_{i}^{2}\right\rangle=[\boldsymbol{\Gamma}]_{i i} \geq\left[\mathbf{I}_{\mathbf{F}}{ }^{-1}\right]_{i i}=[\mathbf{Y}]_{i i}
$$

where $\mathbf{Y}$ is the CRB matrix, $\mathbf{I}_{\mathbf{F}}$ is the Fisher matrix, which is obtained with $\left[\mathbf{I}_{\mathbf{F}}\right]_{i j}=-\left\langle\partial_{z_{i}} \partial_{z_{j}} \ell(\boldsymbol{n})\right\rangle$, and where $\ell(\boldsymbol{n})$ is the log-likelihood and $\boldsymbol{n}=\left(n_{1}, n_{2}, \ldots, n_{M}\right)^{T}$.

If the measures $n_{m}$ are statistically independent, then $\ell(\boldsymbol{n})=$ $\sum_{m=1}^{M} \log \left[P_{m}\left(n_{m}\right)\right]$. The following property is easy to show.

Property 1: The Cramer-Rao bound (CRB) matrix with binary filters is

$$
\mathbf{Y}_{i j}=\left[\mathbf{I}_{\mathbf{F}}^{-1}\right]_{i j}
$$

with the information matrix:

$$
\left[\mathbf{I}_{\mathbf{F}}\right]_{i j}=\sum_{m=1}^{M} \frac{G_{m i} G_{m j}}{\mu_{m}}=\sum_{m=1}^{M} \frac{\tau_{m} g_{m i} g_{m j}}{\sum_{\ell=1}^{Q} g_{m \ell} z_{\ell}}
$$

The proof is analog to the one developed in [11] and is not detailed here. This property shows that the precision is a function of $\tau_{m}, z_{i}$ and of $g_{m i}$ and thus of the binary filters $\mathbf{F}_{m}$.

\section{B. Description of the considered examples}

In the following the results are illustrated with three examples. For these examples, it is assumed that $M=Q=3$ with measuring times $\tau_{m}$ for each filter equal to $\tau$ (i.e. independent of the filter). These examples are considered since they show different correlation coefficients and different intensity ratios between the spectra. Furthermore, $\tau$ is adjusted so that $\tau \sum_{k=1}^{K} S_{i}\left(v_{k}\right)=\alpha_{i}$ with $\alpha_{i}$ values reported in Table 1 . The spectra of the first example, have been generated such that $S_{i}\left(v_{k}\right)=\left|g_{i, k}\right|^{5}$ where the $g_{i, k}(i \in[1,3], k \in[1,50])$ are identically distributed and statistically independent realizations of

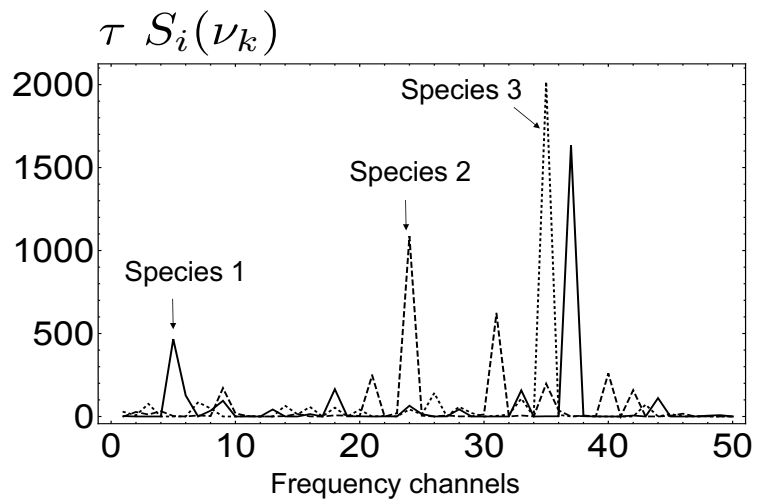

(a)

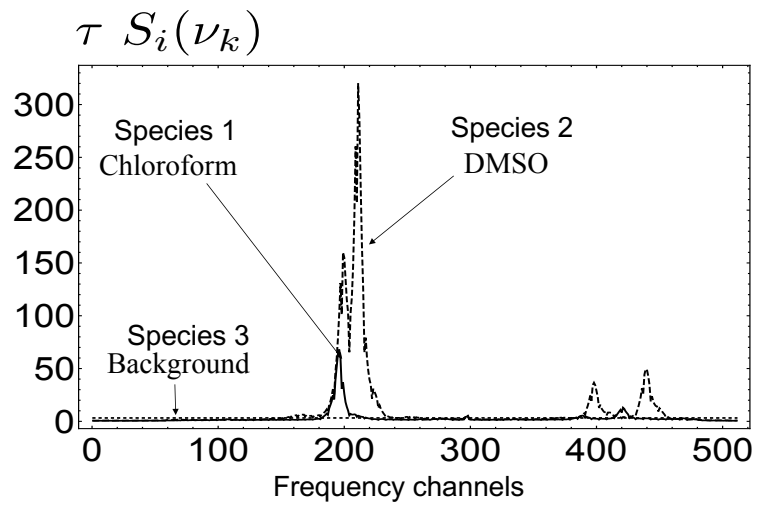

(b)

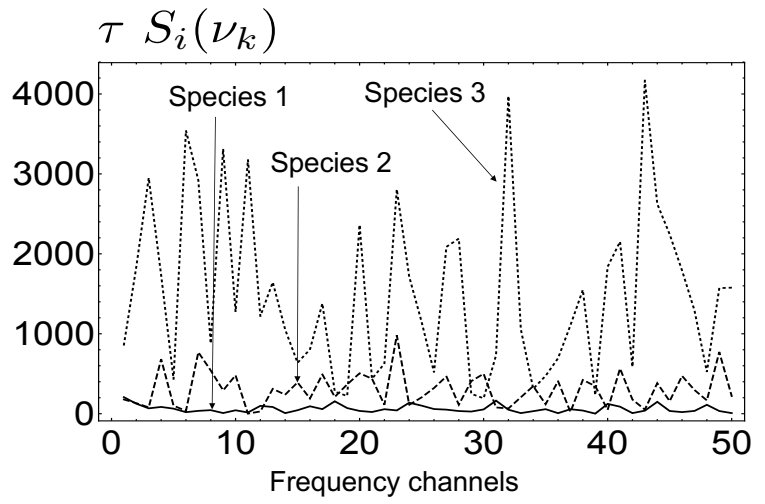

(c)

Fig. 1. Noiseless version of the considered Raman spectra. (a): numerical synthesis on 50 frequency channels of three random spectra (see text for details). (b): Considered models for Raman spectra of Chloroform and Dimethyl sulfoxide (DMSO) acquired on 511 frequency's channels. In this last case, a constant Raman spectrum is added in order to introduce possible background noise. (c) : analogous to (a) but with different spectra (see text for details).

zero mean real Gaussian random variables and normalized so that $\tau \sum_{k=1}^{K} S_{i}\left(v_{k}\right)=\alpha_{i}$ with $K=50$. A noiseless example of such spectra is shown in Fig.1.a. The second example (Fig.1.b) corresponds to the considered models (i.e. noiseless) for Raman spectra of Chloroform and Dimethyl sulfoxide (DMSO) acquired on 511 frequency channels. These spectra are sampled on $K=511$ frequencies and a constant background is also 


\begin{tabular}{|l||l|l|l||}
\hline \hline Ex of & Fig.1.a & Fig.1.b & Fig.1.c \\
\hline$\alpha_{1}$ & 3000. & 1456.3 & 3000. \\
$\alpha_{2}$ & 3000. & 5904.89 & 15000. \\
$\alpha_{3}$ & 3000. & 1638.81 & 75000. \\
\hline$r_{12}$ & 0.0396 & 0.4882 & 0.5602 \\
$r_{23}$ & 0.1656 & 0.3191 & 0.6536 \\
$r_{13}$ & 0.0106 & 0.4074 & 0.6065 \\
\hline \hline
\end{tabular}

Table 1. Values of $\alpha_{i}=\tau \sum_{k=1}^{K} S_{i}\left(v_{k}\right)$ for spectra of Fig. 1. Correlation coefficients correspond to $r_{i j}=$ $\sum_{k=1}^{K}\left[S_{i}\left(v_{k}\right) S_{j}\left(v_{k}\right)\right] / \sqrt{\left(\sum_{k=1}^{K} S_{i}\left(v_{k}\right)^{2}\right)\left(\sum_{k=1}^{K} S_{j}\left(v_{k}\right)^{2}\right)}$.

present in order to introduce possible background noise.

The third example is generated as for the first example but with spectra $S_{i}\left(v_{k}\right)=\left|g_{i, k}\right|$ (see Fig.1.c) normalized so that $\tau \sum_{k=1}^{K} S_{i}\left(v_{k}\right)=\alpha_{i}$ with values reported Table 1. Examples of Figs. 1.a and 1.c have only $K=50$ frequencies to simulate low variation with the frequency channel observed with most real spectra, but the methodology can be applied with any $K$ value larger than $Q$ and $M$. The normalized correlation values between the spectra are also reported in table 1. They show that the considered examples of Figs. 1.a, 1.b and 1.c also correspond to different overlap between the spectra.

\section{Numerical simulation results}

The set $\mathbf{F}$ of the $M$ filters $\mathbf{F}_{m}$ can be determined by minimizing a scalar quantity deduced from $\mathbf{Y}$. Analyzing the trace $\mathcal{E}_{t r}=\operatorname{tr}[\mathbf{Y}]$ of the CRB matrix is interesting since, for unbiased estimators, $\mathcal{E}_{t r}$ is a bound of the minimal value of the mean square error (i.e. of $\operatorname{tr}[\boldsymbol{\Gamma}]$ ). The set $\mathbf{F}$ can be obtained with a simple numerical optimization technique that consists in choosing randomly an $m$ value and a $k$ value and changing the value $F_{m}\left(v_{k}\right)$ into $1-F_{m}\left(v_{k}\right)$ if $\mathcal{E}_{t r}$ is decreased. The process can then be iterated a fixed number of trials. Such a simple approach does not guarantee optimality properties and the convergence can correspond to local minima.

Results of convergence with 50 different random initial conditions for the binary filters of the spectra of Fig.1 are shown in Figs.2, 3 and 4. For these numerical experiments the set $\mathbf{F}$ have been obtained when the proportion coefficients $z_{i}$ are equal to $1 / 3$. The influence of such an arbitrary choice will be discussed in a following section. The number of iterations for each random initialization is $910^{3}$ for the example of Fig.1.a and Fig.1.c and is $910^{4}$ for the example of Fig.1.b. It can be observed that the optimization process does not always lead to the same criterion values and thus to the same filters. An important gain for the CRB can nevertheless be seen after filter optimization in comparison to the values before optimization. Convergence problems for some trials lead to higher CRBs than the minimal value. It can thus be appropriate with such a simple optimization process to perform several optimizations with different initial values and to select the best solution. More sophisticated optimization algorithms could be implemented $[7,8]$ to improve the convergence properties of the method if necessary. However, such a possibility will not be analyzed in this paper since, in the following, it will be imposed that the binary filters are orthogonal.
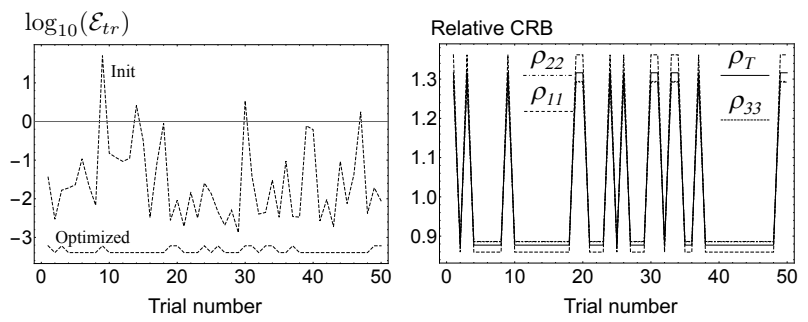

Fig. 2. Results for spectra of Fig. 1.a.

Left: decimal logarithm value of the optimized criterion $\mathcal{E}_{t r}$ obtained for 50 different random initial conditions with $\mathrm{BF}$ determined for $z_{i}=1 / 3$.

Right: Normalized values of the obtained CRB determined with 50 different initial conditions with $\rho_{i i}(n)=\mathrm{Y}_{i i}(n) / \overline{\mathrm{Y}_{i i}}$ and $\rho_{T}(n)=\frac{1}{3} \sum_{i=1}^{3} \rho_{i i}(n)$, where $\overline{Y_{i i}}=\frac{1}{50} \sum_{n=1}^{50} Y_{i i}(n)$ and where $Y_{i i}(n)$ is the obtained value for trial number $n$.
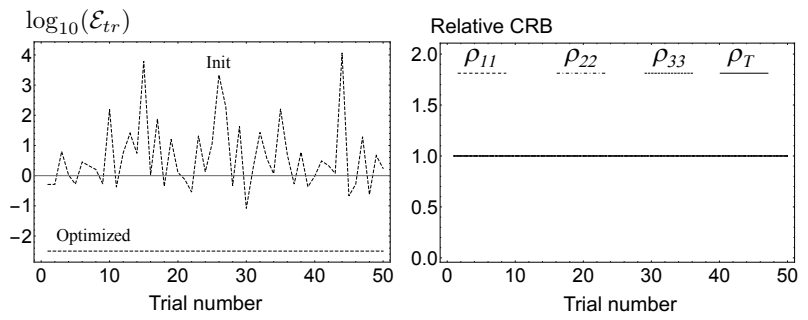

Fig. 3. Same as Fig. 2 but for spectra of Fig. 1.b.
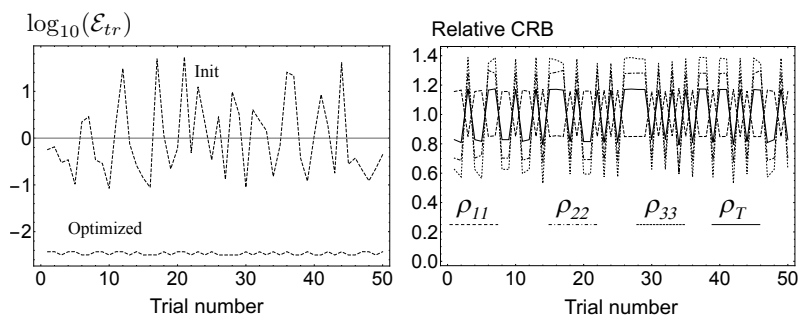

Fig. 4. Same as Fig. 2 but for spectra of Fig. 1.c.

\section{Particular case of $M=Q$}

The particular case for which the number of filters $M$ is equal to the number of known species $Q$ is specially interesting. Indeed, not only it corresponds to the minimal number of needed filters to perform the estimation, but also it allows one to obtain several useful properties. In particular, Property 1 can be simplified in that case into the following corollary.

Corollary 1: When $M=Q$ and if $\mathbf{G}$ is not singular, then

$$
\mathbf{Y}=\mathbf{G}^{-1} \boldsymbol{\Lambda}\left[\mathbf{G}^{T}\right]^{-1}
$$

where the diagonal matrix $\boldsymbol{\Lambda}$ is defined so that $[\boldsymbol{\Lambda}]_{m m}=\mu_{m}$. This equation can be developed, which leads to the following corollary that will be helpful in the following. 
Corollary 2: When $M=Q$ and when $\mathbf{G}$ is non-singular, the CRB $Y_{i i}$ can be written

$$
\mathrm{Y}_{i i}=\sum_{n=1}^{Q} \sum_{m=1}^{Q} \eta_{i m n} \frac{z_{n}}{\tau_{m}}
$$

with

$$
\eta_{i m n}=b_{i m}^{2} g_{m n} \geq 0
$$

where $\boldsymbol{b}=\boldsymbol{g}^{-1}$ with $[\boldsymbol{g}]_{m n}=g_{m n}$ and $[\boldsymbol{b}]_{i m}=b_{i m}$.

This result shows that the CRB $Y_{i i}$ is a linear function of $z_{i}$ when $M=Q$.

\section{E. Efficient estimator when $M=Q$}

It is not sure in general that an unbiased estimator with a variance equal to the CRBs exists. The pseudo-inverse solution, which is analogous to the proposed method of $[4,7,9]$, corresponds to:

$$
\widehat{z}_{l i n}=\left[\mathbf{G}^{T} \mathbf{G}\right]^{-1} \mathbf{G}^{T} \boldsymbol{n}
$$

where it is assumed that $\mathbf{G}^{T} \mathbf{G}$ is non singular. This linear method is unbiased since $\left\langle\widehat{z}_{\text {lin }}\right\rangle=\left[\mathbf{G}^{T} \mathbf{G}\right]^{-1} \mathbf{G}^{T} \boldsymbol{\mu}$ which, with Eq. (6), leads to $\left\langle\widehat{z}_{\text {lin }}\right\rangle=\boldsymbol{z}$.

When $M=Q$ and when $\mathbf{G}$ is non-singular the relation of Eq. (13) simplifies into $\widehat{z}_{\text {lin }}=\mathrm{G}^{-1} \boldsymbol{n}$. Furthermore, the covariance matrix of the estimations is

$$
\Gamma^{(\text {lin })}=\left\langle\delta \widehat{z}_{\text {lin }} \delta \widehat{z}_{\text {lin }}^{T}\right\rangle
$$

where $\delta \widehat{\boldsymbol{z}}_{\text {lin }}=\widehat{\boldsymbol{z}}_{\text {lin }}-\left\langle\widehat{\boldsymbol{z}}_{\text {lin }}\right\rangle$. Since $n_{m}$ are random Poisson variable $\left\langle\delta n_{m}^{2}\right\rangle=\left\langle n_{m}\right\rangle=\mu_{m}$, where $\delta n_{m}=n_{m}-\left\langle n_{m}\right\rangle$. With the diagonal matrix $\Lambda$ defined above, when $M=Q$ and when $\mathbf{G}$ is non singular, the covariance matrix can be written

$$
\Gamma^{(\operatorname{lin})}=\mathbf{G}^{-1} \boldsymbol{\Lambda}\left[\mathbf{G}^{T}\right]^{-1}=\mathbf{Y}
$$

which shows that this estimator is efficient (i.e. unbiased with a variance equal to the $\mathrm{CRB}$ ). It can also be shown that it corresponds to the maximum likelihood estimator (see Appendix A). These results can be summarized in the following property.

Property 2: When $M=Q$ and when $\mathbf{G}$ is non-singular, the linear estimation method

$$
\widehat{z}_{l i n}=\mathrm{G}^{-1} n
$$

is efficient for the estimation of each $z_{i}$, i.e. unbiased with minimum variance. Furthermore, this solution corresponds to the maximum likelihood estimator.

Furthermore, since $\Gamma^{(\text {lin })}=\mathbf{Y}$, this result shows that minimizing the mean square error [7-9] is efficient with Poisson noise model for the considered application when $M=Q$. In other words, it is equivalent to design the binary filters by minimizing either the trace of the CRB matrix or the mean square error when $M=Q$.

\section{F. Bounds with unequal measuring times when $M=Q$}

It has been mentioned in $[7,8]$ that the time $\tau_{m}$ can be chosen in order to minimize the mean square error of the $z_{i}$ estimations. It is possible to obtain a bound on the possible gain that can be expected for a fixed set $\mathbf{F}$ of binary filters as shown by the following property (shown in Appendix B).

Property 3: Let $\mathrm{Y}_{i i}^{\left(\tau_{0}\right)}$ denote the CRBs with BF method with equal measuring time $\tau_{0}=T / Q$ when $M=Q$ and $Y_{i i}^{(\bar{\tau})}$ the CRBs obtained with measuring times $\bar{\tau}=\left(\tau_{1}, . ., \tau_{Q}\right)^{T}$ with $\sum_{i=1}^{Q} \tau_{i}=T$. Then,

$$
\mathrm{Y}_{i i}^{(\bar{\tau})} \geq \frac{1}{Q} \mathrm{Y}_{i i}^{\left(\tau_{0}\right)}
$$

Thus, optimizing the measuring times cannot lead to a decrease of the CRB larger than a factor $Q$ in comparison to the result obtained with equal measuring times. In other words, the gain is bounded by a factor $Q$ but it can nevertheless be observed that if $\tau_{m} \rightarrow 0$ then $\mathrm{Y}_{i i}^{(\bar{\tau})} \rightarrow \infty$ which shows that the loss is not bounded if an inappropriate choice of the $\tau_{m}$ is done.

\section{G. Case of upper bounded measuring times when $M=Q$}

The situation is different if the constraint corresponds only to upper bounded measuring time $\tau_{m}$. Such a situation appears if the filters are applied in parallel. Although practically difficult to implement, this situation is in principle possible with orthogonal binary filters. In that case the following property shows that no gain can be expected with $\tau_{m}$ optimization when $M=Q$.

Property 4: When $M=Q$ and when the constraint $\tau_{m} \leq \tau_{0}$ is imposed, the minimal CRB values are obtained with $\tau_{m}=\tau_{0}$.

Indeed, Corollary 2 leads to (see Appendix B)

$$
\mathrm{Y}_{i i}^{(\bar{\tau})}=\sum_{m=1}^{Q} \frac{\beta_{i m}}{\tau_{m}} \geq \sum_{m=1}^{Q} \frac{\beta_{i m}}{\tau_{0}}
$$

since $\beta_{i m} \geq 0$. The minimal CRB values are thus obtained with equal measuring times $\tau_{m}=\tau_{0}$.

\section{H. Discussion when $M=Q$}

The comparison between systems for which the binary filters are applied sequentially or in parallel is interesting. Indeed, let $T$ be the total measuring time. With $M \mathrm{BF}$ applied sequentially the constraint is $\sum_{m=1}^{M} \tau_{m}=T$. With $M$ OBF applied in parallel the constraint is $\tau_{m} \leq T$ and the optimal solution (i.e. that minimizes the CRB) is $\tau_{m}=T$. However, $Y_{i i}^{(T / Q)}=Q Y_{i i}^{(T)}$ which is another illustration of the result of property 4 that there is no possible gain when OBF are applied in parallel with optimization of the measuring times. Since in the following, OBF will be assumed implemented in parallel when compared to full Raman method, the measuring times will be considered equal. However, the results obtained below do not need that the binary filters are implemented in parallel. If they are implemented sequentially, the CRB have thus simply to be multiplied by $M$ for the same total measuring time. 


\section{COMPARISON WITH FULL RAMAN METHOD}

\section{A. Cramer-Rao bound of the full Raman method}

The CRB for estimating the $z_{i}$ coefficients with full Raman methods has been determined in [11]. The most favorable situation for the full Raman technique is obtained when the noise is photon limited. In that case, the CRB is simple and leads to the following corollary.

Corollary 3: The information matrix with full Raman method is

$$
\left[\mathbf{I}_{\mathbf{F}}{ }^{(F)}\right]_{i j}=\tau \sum_{k=1}^{K} \frac{S_{i}\left(v_{k}\right) S_{j}\left(v_{k}\right)}{S\left(v_{k}\right)}
$$

An extension of this result in the presence of detector noise and background noise is discussed in Appendix C. This corollary also shows that the CRB is inversely proportional to the measuring time (which is also the case for BF and OBF with equal measuring times for each filter).

\section{B. Orthogonal binary filters}

Orthogonal binary filters (OBF) are defined so that $F_{m}\left(v_{k}\right)=0$ or 1 and $\sum_{m=1}^{M} F_{m}\left(v_{k}\right) \leq 1$. In other words, for each frequency $v_{k}$ only at most one of the binary filters satisfies $F_{m}\left(v_{k}\right)=1$. Although difficult to realize, such OBF allows one to perform the measurements with BF in parallel.

The BF optimization technique can be adapted to design the set $\mathbf{F}$ of the OBF. For each randomly chosen $k$, a new set of the $M$ values $F_{m}\left(v_{k}\right)$ that satisfies $F_{m}\left(v_{k}\right)=0$ or 1 and $\sum_{m=1}^{M} F_{m}\left(v_{k}\right) \leq$ 1 is chosen randomly. This new set of values is accepted if $\mathcal{E}_{t r}$ is decreased. Results of convergence with 50 different random initial OBF for the spectra of Fig.1 are shown in Figs.5, 6 and 7. The number of iterations for each random initialization is $910^{3}$ for the example of Fig.1.a and Fig.1.c and is $910^{4}$ for the example of Fig.1.b. It is interesting to notice on these examples that OBF do not lead to an important increase of the CRB in comparison to the ones of the BF.
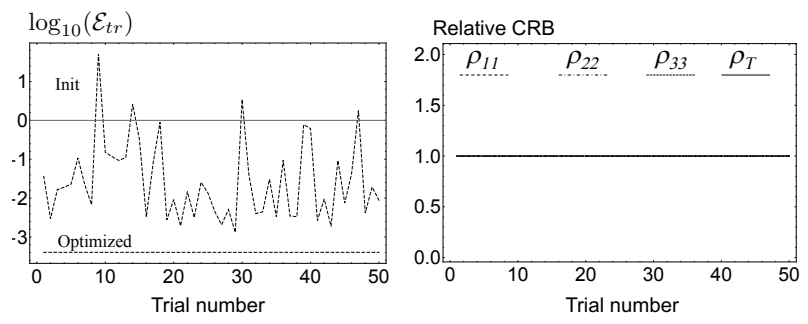

Fig. 5. Same as Fig. 2 but with OBF.
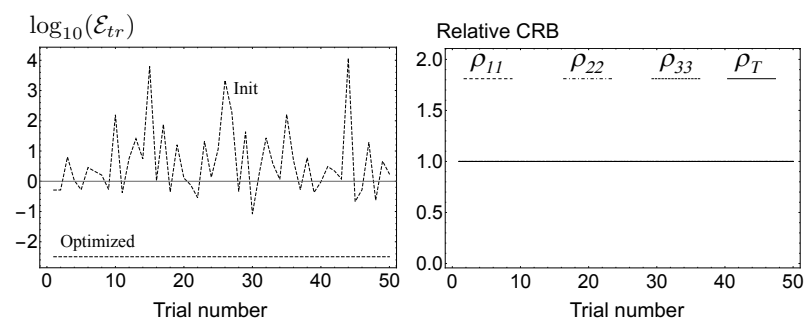

Fig. 6. Same as Fig. 3 but with OBF.
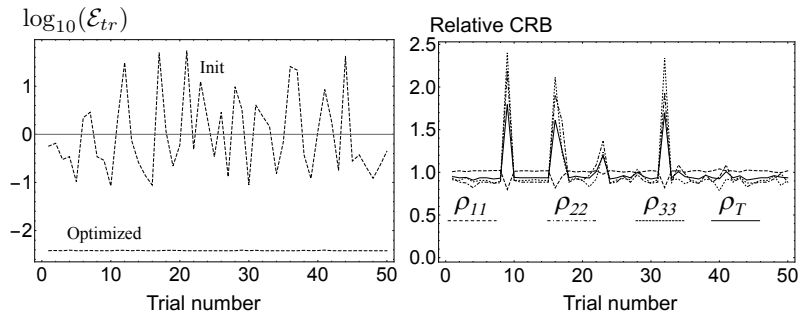

Fig. 7. Same as Fig. 4 but with OBF.

\section{Comparison of orthogonal binary filters with full Raman method}

When the measuring times for each filter are equal, it is possible to compare the OBF method to the full spectral Raman measurements (FSR). Indeed, when the filters are orthogonal the probability law of $n_{m}$ with $m=1, . ., M$ is equal to the one of $n_{m}^{\prime}=\sum_{k=1}^{K} F_{m}\left(v_{k}\right) N_{\tau}\left(v_{k}\right)$ where $N_{\tau}\left(v_{k}\right)$ is the number of photons that would have been obtained with the FSR approach. In other words, the $n_{m}^{\prime}$ determined with a numerical binary filtering of photons detected with a full Raman approach are independent if the binary filters are orthogonal. Thus, orthogonality of the filters assures that the $n_{m}^{\prime}$ are statistically independent and the CRB with either the $n_{m}$ or the $n_{m}^{\prime}$ are still given by Eq. (9). From an information theory point of view, the OBF approach is thus equivalent to perform the estimation on the binary numerically filtered signal obtained after the photons have been detected with a full Raman approach (FSR). OBF are then a subset of possible algorithms with FSR, and thus, the CRBs with OBF cannot be smaller than the ones with FSR. These considerations lead to the following property.

Property 5: Let $Y_{i i}^{\left(F, \tau_{0}\right)}$ denote the CRB with Full Raman measurements with measuring time $\tau_{0}$ and $Y_{i i}^{\left(\tau_{0}\right)}$ the optimal CRBs obtained with OBF with equal measuring times $\tau_{0}$, the CRBs satisfy

$$
\mathrm{Y}_{i i}^{\left(\tau_{0}\right)} \geq \mathrm{Y}_{i i}^{\left(F, \tau_{0}\right)}
$$

Obviously this property implies that $\mathcal{E}_{t r}=\sum_{i=1}^{Q} \mathrm{Y}_{i i}^{\left(\tau_{0}\right)} \geq$ $\mathcal{E}_{t r}^{(F)}=\sum_{i=1}^{Q} Y_{i i}^{\left(F, \tau_{0}\right)}$. It is interesting to emphasize that this property is not shown for non orthogonal binary filters. Indeed, in that latter case (i.e. with BF), the random values $n_{m}$ with $m=1, . ., M$, obtained with the binary filters are statistically independent since they result from independent Poisson measurements. However, the random variables $n_{m}^{\prime}=$ $\sum_{k=1}^{K} F_{m}\left(v_{k}\right) N_{\tau}\left(v_{k}\right)$, where $N_{\tau}\left(v_{k}\right)$ is the number of photons that would have been obtained with the FSR approach, are not statically independent if the values $F_{m}\left(v_{k}\right)$ are non zero for several $m$ values.

\section{Robustness to the chosen proportion for OBF synthesis}

In the above examples the BF or OBF have been determined by minimizing $\mathcal{E}_{t r}$ when the proportions are $z_{i}^{(d)}=1 / 3$. Since, the proportion values are unknown, it is interesting to analyze the robustness of the estimation method when the actual proportions $z_{i}$ are different to $z_{i}^{(d)}$. The CRB of the Full Spectrum Raman (FSR), which can be easily determined for each $z_{i}$ possible values, is useful for that purpose. The CRBs obtained 
with OBF and with FSR and the ratios of their square-roots are shown in Figs. 8 and 9 as a function of $z_{1}$ and $z_{2}$ (assuming $z_{3}=1-z_{1}-z_{2}$ ) for both examples of Fig.1.a and Fig.1.b. It can be seen for these examples that these ratios are close to 1 , although differences appear on the boundary of the triangle. Thus, with examples of Figs. 1.a and 1.b, designing OBF with $\mathcal{E}_{\text {tr }}$ calculated for $z_{i}^{(d)}=1 / 3$ does not lead to an important loss of performance for a large set of $z_{i}$ values. Obviously, these examples are not representative of all the possible situations as shown with Fig.10 that reports an analogous analysis for example of Fig.1.c. It clearly appears that the loss of performance is higher than for the previous examples but it is still small since it is of the order of $50 \%$ (for the standard deviation). For this example the number of measured photons is larger than for examples of Figs. 1.a and 1.b but the CRBs are still of the order $10^{-2}$. Higher losses can probably appear for other examples and the proposed methodology allows one to precisely quantify them.
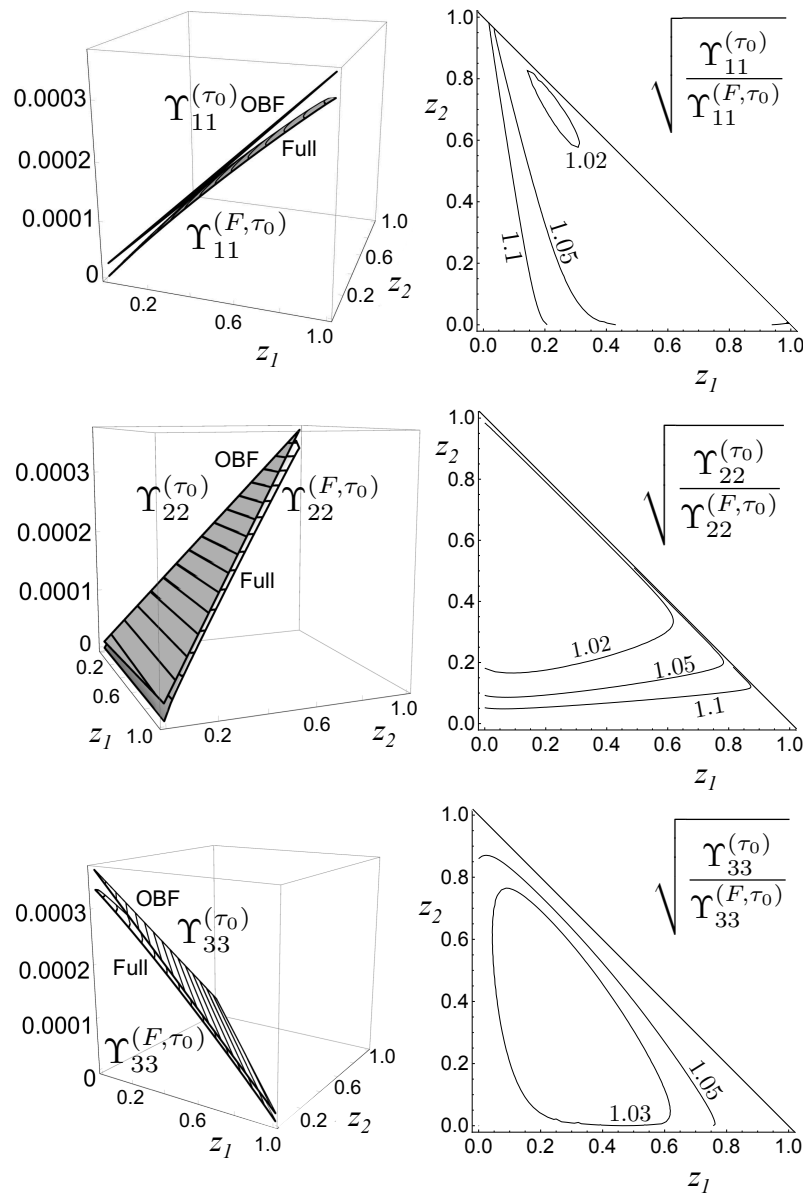

Fig. 8. Results for spectra of Fig.1.a.

Left: CRB obtained with orthogonal binary filters (OBF) designed for $z_{i}^{(d)}=1 / 3$ and with the full Raman spectrum as a function of $z_{1}$ and $z_{2}\left(z_{3}=1-z_{1}-z_{2}\right)$.

Right: Ratio of the square-root of the CRB obtained with orthogonal binary filters (OBF) designed for $z_{i}^{(d)}=1 / 3$ with the square-root of the CRB using the full Raman spectrum as a function of $z_{1}$ and $z_{2}$.

It has to be noticed that the comparison has been done assuming that the $M$ measurements with OBFs are performed in
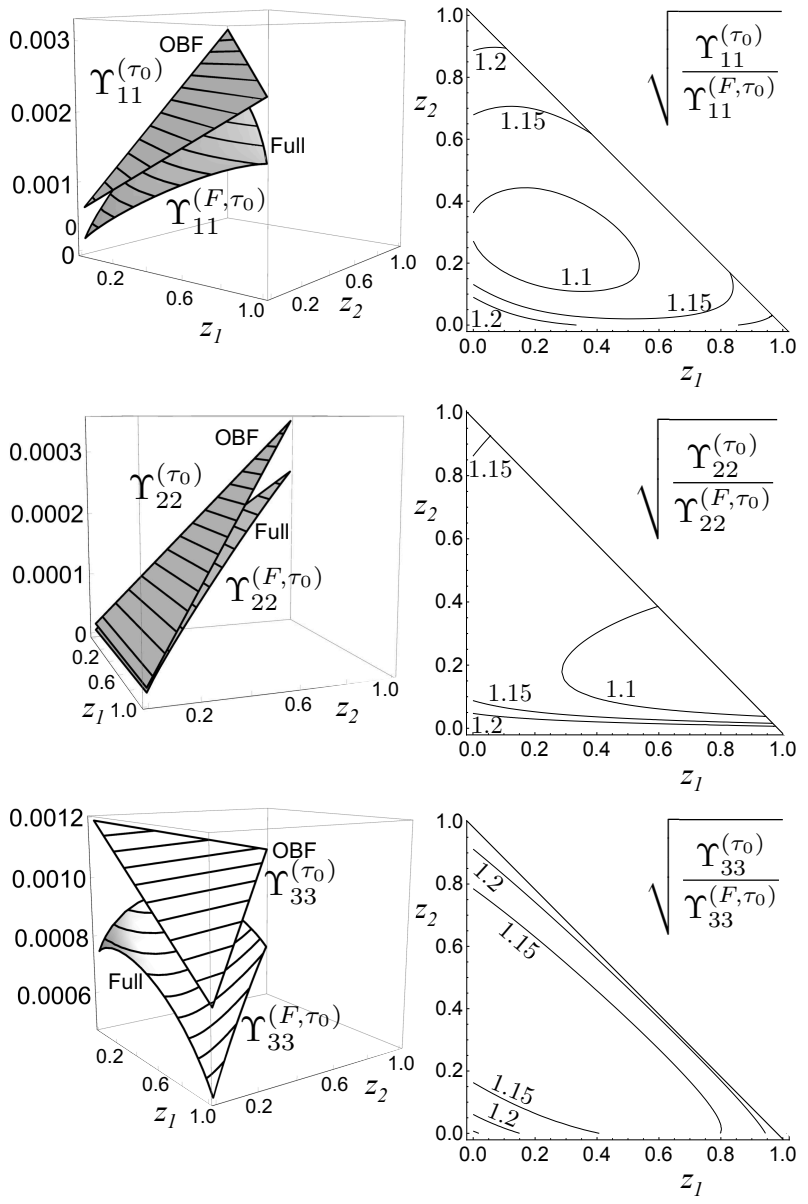

Fig. 9. The same as for Fig.8 but for spectra of Fig.1.b.

parallel. If the measurements are performed sequentially, equivalent CRBs are obtained only if the total measuring time for $\mathrm{OBF}$ is $M$ times the one of FSR.

With this assumption of parallel measurements, several remarks can be mentioned when the CRBs for OBF and for the full Raman method are approximately equal. Indeed, in that case, using only few binary combinations of the Raman spectrum does not degrade significantly the precision in retrieving the proportions $z_{i}$. Firstly, minimizing the sum of the CRBs (or equivalently the mean square error) for OBF optimization is approximately efficient to minimize the CRB for each species although they can be different for each species. Secondly, using more OBF than the number of species will not decrease significantly the CRB. Thirdly, specializing the OBF for each $z_{i}$ value may not be necessary for a large set of $z_{i}$ values.

However, there is no proof that the CRBs for OBF and for the full Raman method are always approximately equal and it is not the case as shown with the example of Fig.1.c. It is thus interesting to analyze more precisely the influence of the choice of the proportion $z_{i}^{(d)}$ considered for the OBF synthesis. 

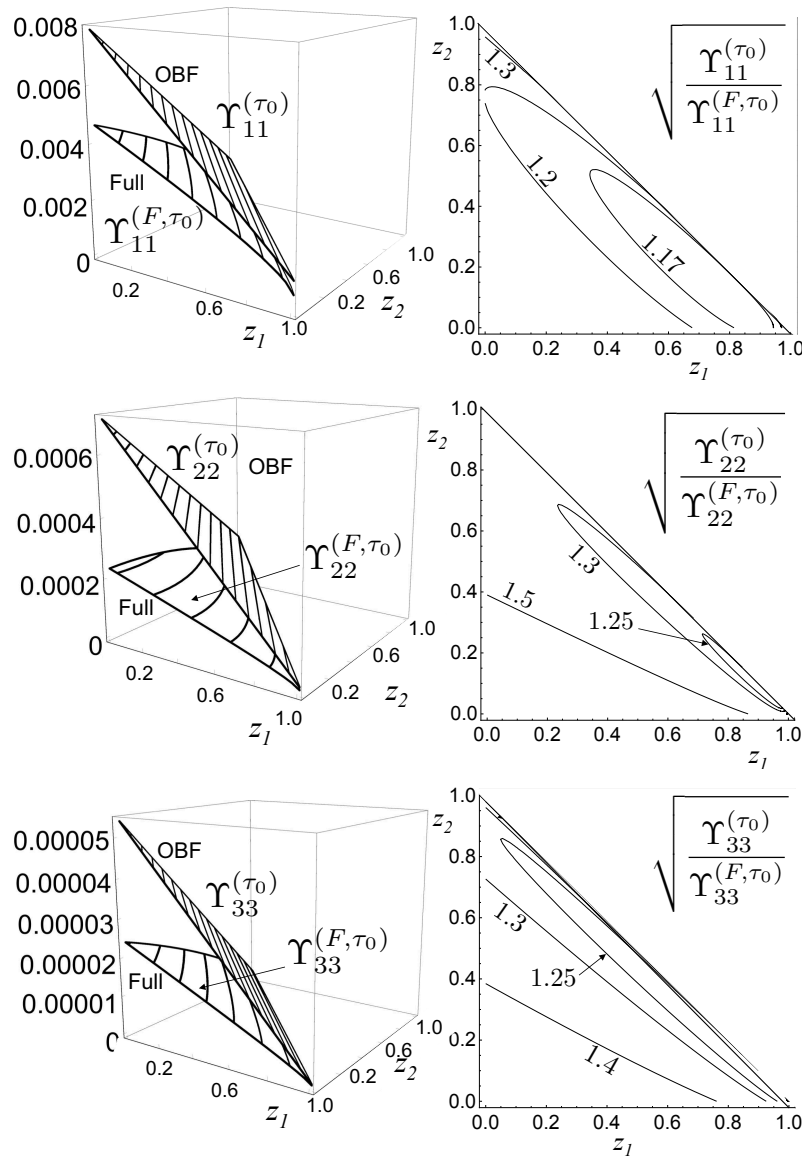

Fig. 10. The same as for Fig.8 but for spectra of Fig.1.c.

\section{INFLUENCE OF THE CONSIDERED PROPORTION FOR OBF DESIGN}

\section{A. Problem}

In the above analyses, the proportion $z$ for which the BF (or $\mathrm{OBF}$ ) filters have been designed was chosen a priori. In the following, two approaches are considered in order to optimize this choice. With the first one, the mean average value of the criterion in the set $\mathcal{T}$ (i.e. so that $\sum_{i=1}^{Q} z_{i}=1$ with $z_{i} \geq 0$ ) is minimized when the probability density function of the $z_{i}$ satisfies symmetry properties. The second approach is a minimax technique that consists in minimizing the maximal value in $\mathcal{T}$. Although the results presented below are developed for $\mathrm{BF}$ when $M=Q$ they will be illustrated for OBF with the same hypothesis.

Eq. 11 of corollary 2 can be written $Y_{i i}=\sum_{n=1}^{Q} \gamma_{i n} z_{n}$ with $\gamma_{i n}=\sum_{m=1}^{Q} \frac{b_{i m}^{2} g_{m n}}{\tau_{m}} \geq 0$. Let $\mathbf{F}(\boldsymbol{x})$ be the set of BF $\mathbf{F}_{m}$ that minimizes $\mathcal{E}_{t r}$ for proportion $x$. Then, mentioning explicitly the proportion $x$ for which the BF have been optimized and the proportion $z$ for which the BF are used, the CRB can be written

$$
\mathrm{Y}_{i i}(\boldsymbol{z}, \mathbf{F}(\boldsymbol{x}))=\sum_{n=1}^{Q} \gamma_{i n}(\boldsymbol{x}) z_{n}
$$

Furthermore, $\mathcal{E}_{t r}(\boldsymbol{z}, \mathbf{F}(\boldsymbol{x}))=\sum_{i=1}^{Q} \mathrm{Y}_{i i}(\boldsymbol{z}, \mathbf{F}(\boldsymbol{x}))$ and thus

$$
\mathcal{E}_{\text {tr }}(\boldsymbol{z}, \mathbf{F}(\boldsymbol{x}))=\sum_{n=1}^{Q} z_{n} \mathcal{E}_{n}(\boldsymbol{x})
$$

where $\mathcal{E}_{n}(x)=\sum_{i=1}^{Q} \gamma_{i n}(x)$. This result clearly shows that $\mathcal{E}_{t r}(\boldsymbol{z}, \mathbf{F}(\boldsymbol{x}))$ is a linear function of $\boldsymbol{z}$, which allows one to show several properties.

\section{B. Minimizing the mean value of the mean square error}

When the BF are synthesized for $z_{i}=1 / Q$ the CRB of the estimation of $z_{i}$ is bounded in $\mathcal{D}$, where $\mathcal{D}$ is the set of $z$ values that satisfy $0 \leq z_{i} \leq 1$, as shown by the following property (see Appendix D).

Property 6: When $M=Q$ and when $g$ is non-singular, the CRB for $\mathcal{D}$ satisfies

$$
\mathrm{Y}_{i i}\left(\boldsymbol{z}, \mathbf{F}\left(x_{Q}\right)\right) \leq Q \mathrm{Y}_{i i}\left(x_{Q}, \mathbf{F}\left(x_{Q}\right)\right)
$$

where $x_{Q}=(1 / Q, \ldots, 1 / Q)^{T}$.

Thus choosing equal proportions $x_{Q}$ for filter synthesis may lead in $\mathcal{D}$ to an increase of the CRB bounded by a factor $Q$.

When no a priori information is available on the $z_{i}$ values, it can be interesting to consider a probability density function (pdf) of the $z_{i}$ in $\mathcal{T}$ that is invariant by the permutation of the $z_{i}$. In that case the following property can be shown (see Appendix E).

Property 7: When $M=Q$ and when $g$ is non-singular, the average value of $\mathcal{E}_{t r}(\boldsymbol{z}, \mathbf{F}(\boldsymbol{x}))$ over $\boldsymbol{z}$ in $\mathcal{T}$ with a probability density function of $z$ invariant by permutation of the $z_{i}$, is equal to its value for $z_{i}=1 / Q$.

With such symmetrical pdf, determining BF or OBF for $z_{i}=$ $1 / Q$ is equivalent to minimize the mean value of the $C R B$ in $\mathcal{T}$. However, if the constraint $\sum_{i=1}^{Q} z_{i}=1$ is not satisfied this property will generally not be valid.

\section{Minimizing the maximal value of the mean square error}

Minimizing the average value of $\mathcal{E}_{t r}(\boldsymbol{z}, \mathbf{F}(\boldsymbol{x}))$ is not the only possible strategy. For example it can be interesting to develop methods that minimize the maximal value of $\mathcal{E}_{t r}(\boldsymbol{z}, \mathbf{F}(x))$ for $\boldsymbol{z} \in \mathcal{T}$. Such a minimax strategy does necessary lead to the solution $z_{i}=1 / Q$. The minimax solution (i.e. the value of $x$ that minimizes the maximal value of $\mathcal{E}_{t r}(\boldsymbol{z}, \mathbf{F}(x))$ for $\left.z \in \mathcal{T}\right)$ is dependent of the considered problem and thus of spectra $S_{i}\left(v_{k}\right)$.

The minimax algorithm is simple to implement with the properties shown above. Indeed, Eq. 22 leads to

$$
\max _{\boldsymbol{z} \in \mathcal{T}} \mathcal{E}_{t r}(\boldsymbol{z}, \mathbf{F}(\boldsymbol{x}))=\max _{n} \mathcal{E}_{n}(\boldsymbol{x})
$$

Thus for each $x$ values, the set $\mathbf{F}(\boldsymbol{x})$ that minimizes $\mathcal{E}_{t r}(x, \mathbf{F})$ can be determined with the method described in the previous section. The values $\mathcal{E}_{n}(x)$ are thus obtained for each tested $x$ value and it is straightforward to get $\max _{n} \mathcal{E}_{n}(x)$. Both values $\mathcal{E}_{t r}(\boldsymbol{z}, \mathbf{F}(\boldsymbol{z}))$ and $\max _{\boldsymbol{x} \in \mathcal{T}} \mathcal{E}_{t r}(\boldsymbol{x}, \mathbf{F}(\boldsymbol{z}))$ are shown in Fig.11 for the considered examples of this article for OBF synthesis. For that purpose the $z_{i}$ are sampled on 21 values between 0.003333 .. and 0.993333....

These results show that designing OBF for one proportion may not be a robust approach. Indeed, if the filter is designed for proportion $x_{d}$, the value of the optimized criterion $\mathcal{E}_{t r}\left(x_{d}, \mathbf{F}\left(x_{d}\right)\right)$ can be small in comparison to its maximal value in $\mathcal{T}$. It can also be seen on the considered examples, that $\max _{\boldsymbol{z} \in \mathcal{T}} \mathcal{E}_{t r}(\boldsymbol{z}, \mathbf{F}(\boldsymbol{x}))$ is minimal for proportion $\boldsymbol{x}$ near the one that maximizes $\mathcal{E}_{t r}(\boldsymbol{x}, \mathbf{F}(\boldsymbol{x}))$. 
The above analysis is based on the assumption that $z \in \mathcal{T}$ that allows one to get simple representations and algorithms. However, $\sum_{i=1}^{Q} z_{i}=1$ may not be satisfied for some species mixing. The minimax strategy could nevertheless be adapted to more complex situations with a numerical search of the maximal value of $\mathcal{E}_{t r}(\boldsymbol{z}, \mathbf{F}(\boldsymbol{x}))$.
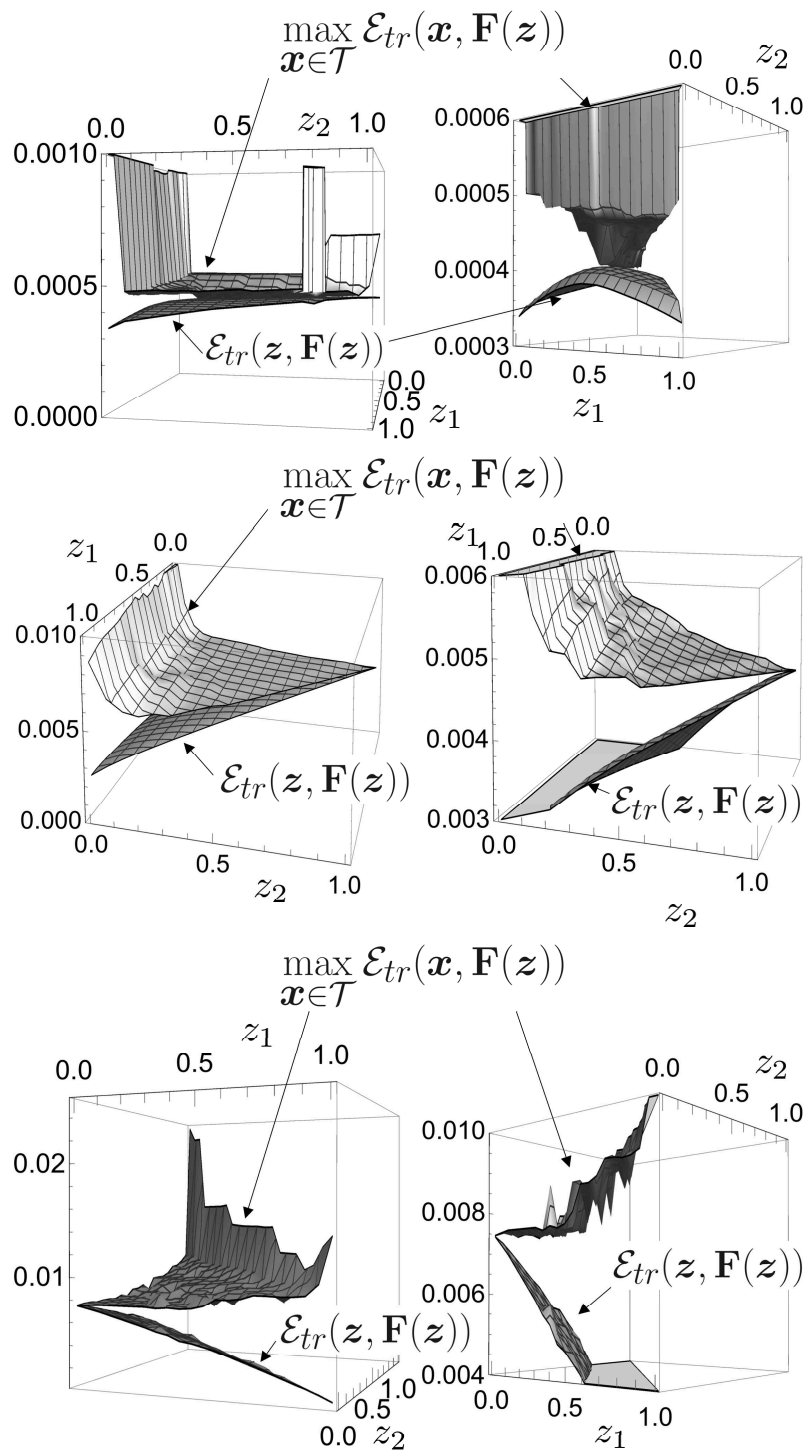

Fig. 11. Lower surfaces: $\mathcal{E}_{t r}(\boldsymbol{z}, \mathbf{F}(\boldsymbol{z}))$ and upper surfaces: $\max _{\boldsymbol{x} \in \mathcal{T}} \mathcal{E}_{\text {tr }}(\boldsymbol{x}, \mathbf{F}(\boldsymbol{z}))$.

First Line: example of fig1.a.

Second Line: example of fig1.b.

Third Line: example of fig1.c.

\section{CONCLUSION AND PERSPECTIVES}

In conclusion, binary filtering of Raman spectra and Full Raman spectrum method have been compared for proportion estimation of mixing of several species [4,7] when the measured spectrum is a linear function of the elementary species. A particular attention has also been devoted to the case for which the number of filters is equal to the number of unknown species and to the mixing of three species. Furthermore, the analysis has also been conducted for orthogonal binary filters, which allows one to perform the measurements in parallel. Bounds on the Cramer-Rao bounds have been provided when the measuring times are optimized or when the considered proportions for binary filter synthesis are not optimized. For parallel implementation, it is not relevant to optimize the measuring times but analyzing the considered proportions for binary filter synthesis can be important. Two strategies for an appropriate choice of this considered proportions have been analyzed for the binary filter synthesis. In particular, the analysis of a minimax strategy has shown that non robust solutions can be obtained when these proportions are not chosen carefully. The methodology described in this article has been illustrated with several particular examples, but it can be easily applied to each new mixing of species with known Raman spectra.

Several perspectives exist to this work. Numerical analysis should be performed for mixing of more than three species since it can be expected that, for a fixed number of Raman spectrum frequencies, the efficiency of binary filtering can decrease [7] and more sophisticated filter optimization algorithms may be required [8]. Other criterion than the ones optimized in this paper could also be considered. More interesting, for both a theoretical and experimental point of view, would be the generalization of the analysis to situations for which the relation between the total spectrum and the elementary spectra is not a linear function. The CRB is an interesting concept to investigate such situations. However, the most interesting perspective is probably to implement the proposed method experimentally for practical applications with a precision close to the CramerRao Bound. In particular, since estimation of the proportion coefficients $z_{i}$ is only based on the inversion of the matrix $\mathbf{G}$ that can be measured in situ, simple calibration techniques could be implemented.

\section{FUNDING INFORMATION}

This project has received funding from the European Union's Horizon 2020 research and innovation programme under the Marie Sklodowska-Curie grant agreement No713750. This work has been carried out thanks to the financial support of the Regional Council of Provence-Alpes-Côte d'Azur and of the A*MIDEX project (ANR-11-IDEX-0001-02) funded by the "Investissements d'Avenir" French Government program, managed by the French National Research Agency (ANR), of the France Bio Imaging project (ANR-10-INSB-04-01), of the France Life Imaging project (ANR-11-INSB-0006), of the Centre National de la Recherche Scientifique (CNRS): "Défi ImagIn" grant CODIM and "Ingénieurie pour la santé" PEPS INSIS and of the Institut National de la Santé et de la Recherche Médicale (INSERM: PC201508). H.B. de Aguiar is supported by LabEX ENSICFP: ANR-10-LABX-0010/ANR-10-IDEX-0001-02 PSL.

\section{A. APPENDIX A.}

The maximum likelihood estimate of $\mu_{m}$ is $\widehat{\mu}_{m}=n_{m}$. Thus due to the invariance property of the maximum likelihood estimation [12], the maximum likelihood estimation $\widehat{z}_{i}$ of $z_{i}$ satisfies $\sum_{i} G_{m i} \widehat{z}_{i}=\widehat{\mu}_{m}$. 


\section{B. APPENDIX B.}

Corollary 2 leads to $Y_{i i}^{(\bar{\tau})}=\sum_{m=1}^{Q} \frac{\beta_{i m}}{\tau_{m}}$ with $\beta_{i m}=$ $b_{i m}^{2}\left(\sum_{n=1}^{Q} g_{m n} z_{n}\right)$. Thus $\beta_{i m} \geq 0$ and

$$
\mathrm{Y}_{i i}^{(\bar{\tau})}=\sum_{m=1}^{Q} \frac{\beta_{i m}}{\tau_{0}} \frac{\tau_{0}}{\tau_{m}} \geq \sum_{m=1}^{Q} \frac{\beta_{i m}}{\tau_{0}} \min _{m}\left[\frac{\tau_{0}}{\tau_{m}}\right]
$$

However $\mathrm{Y}_{i i}^{\left(\tau_{0}\right)}=\sum_{m=1}^{Q} \frac{\beta_{i m}}{\tau_{0}}$ and $\min _{m}\left[\frac{\tau_{0}}{\tau_{m}}\right] \geq \frac{1}{Q}$ and thus

$$
\mathrm{Y}_{i i}^{(\bar{\tau})} \geq \frac{1}{Q} \mathrm{Y}_{i i}^{\left(\tau_{0}\right)}
$$

\section{APPENDIX C.}

Detector and background noise can also be taken into account. Since detector noise is generally described with an additive Gaussian probability density function of zero mean while background noise can be considered as Poisson distributed, several approximations can be considered to determine an approximation of the CRB (see also [11]). At sufficiently high flux, Poisson distribution can be approximated with a Gaussian distribution with variance equal to its mean which leads to

$$
\begin{aligned}
P(n(v))= & \frac{1}{\sqrt{2 \pi\left(\sigma_{d}^{2}(v)+B(v)+S(v)\right)}} \\
& \times \exp \left(-\frac{[n(v)-B(v)-S(v)]^{2}}{2\left[\sigma_{d}^{2}(v)+B(v)+S(v)\right]}\right)
\end{aligned}
$$

where $\sigma_{d}^{2}(v)$ is the spectral density power of the additive noise and $B(v)$ is a background noise spectrum. Assuming $\sigma_{d}^{2}\left(v_{k}\right)+$ $B\left(v_{k}\right)+S\left(v_{k}\right) \gg 1$ leads to the information matrix:

$$
\left[\mathbf{I}_{\mathbf{F}}{ }^{(F)}\right]_{i j} \simeq \tau \sum_{k=1}^{K} \frac{S_{i}\left(v_{k}\right) S_{j}\left(v_{k}\right)}{\sigma_{d}^{2}\left(v_{k}\right)+B\left(v_{k}\right)+S\left(v_{k}\right)}
$$

This expression is identical to Eq. (19) when $\sigma_{d}^{2}(v)=B(v)=$ 0 . This approach can also be applied to compressed measurements which, when $M=Q$ and when $\mathbf{G}$ is non singular, leads to the covariance matrix

$$
\boldsymbol{\Gamma}^{(\operatorname{lin})}=\mathbf{G}^{-1} \boldsymbol{\Lambda}_{B, d}\left[\mathbf{G}^{T}\right]^{-1}
$$

where $\Lambda_{B, d}$ is the diagonal matrix with diagonal elements $\left[\boldsymbol{\Lambda}_{B, d}\right]_{m m}=\mu_{m}+\tau_{m} \sum_{k=1}^{K} F_{m}\left(v_{k}\right) B\left(v_{k}\right)+\sigma_{m}^{2}$ where $\sigma_{m}^{2}$ is the variance of the detector additive noise of the measurement with filter $\mathbf{F}_{m}$.

\section{APPENDIX D.}

It has been shown that $Y_{i i}\left(\boldsymbol{z}, \mathbf{F}\left(\boldsymbol{x}_{Q}\right)\right)=\sum_{n=1}^{Q} \gamma_{i n}\left(x_{Q}\right) z_{n}$ with $\gamma_{i n}\left(x_{Q}\right)=\sum_{m=1}^{Q} \frac{b_{i m}^{2} g_{m n}}{\tau_{m}} \geq 0$. Thus, in $\mathcal{D}$, the CRB satisfies $\mathrm{Y}_{i i}\left(\boldsymbol{z}, \mathbf{F}\left(\boldsymbol{x}_{Q}\right)\right) \leq \sum_{n=1}^{Q} \gamma_{i n}\left(\boldsymbol{x}_{Q}\right)$. Furthermore, $\mathrm{Y}_{i i}\left(\boldsymbol{x}_{Q}, \mathbf{F}\left(\boldsymbol{x}_{Q}\right)\right)=$ $\frac{1}{Q} \sum_{n=1}^{Q} \gamma_{i n}\left(x_{Q}\right)$ that leads to the result of property 6 .

\section{E. APPENDIX E.}

Let us consider a probability density function $P(z)$ that is invariant to $z_{i}$ permutations. Thus $P(\boldsymbol{z})=P\left(\boldsymbol{z}^{\prime}\right)$, where $\boldsymbol{z}^{\prime}$ is obtained by permutation of the $z_{i}$ coordinates of $z$. Eq.22 leads to the average value

$$
\overline{\mathcal{E}_{t r}(\boldsymbol{z}, \mathbf{F}(\boldsymbol{x}))}=\sum_{n=1}^{Q} \mathcal{E}_{n}(\boldsymbol{x}) \int \ldots \int z_{n} P(\boldsymbol{z}) \prod_{j=1}^{Q} d z_{j}
$$

Since $P(z)$ is assumed invariant to $z_{i}$ permutations, $\int \ldots \int z_{n} P(z) \prod_{j=1}^{Q} d z_{j}=a$, which is independent of $n$. Furthermore, $\sum_{n=1}^{Q} z_{n}=1$ implies $\overline{\sum_{n=1}^{Q} z_{n}}=1=Q a$ and thus $a=1 / Q$, which ends the proof.

\section{REFERENCES}

1. DeVerse, R. A., Hammaker, R. M. and Fateley, W. G., "Realization of the Hadamard Multiplex Advantage Using a Programmable Optical Mask in a Dispersive Flat-Field Near-Infrared Spectrometer," Appl. Spectrosc., 54, 1751-1758, (2000).

2. Uzunbajakava, N.; de Peinder, P., Hoof, G. W. and van Gogh, A. T., "Low-cost spectroscopy with a variable multivariate optical element," Analytical Chemistry, 78, 7302-7308, (2006).

3. Quyen, N.T., Jouan, M. D., Dao, N. Q., Da Silva, E. and Phuong D. A. "New Raman spectrometer using a digital micromirror device and a photomultiplier tube detector for rapid on-line industrial analysis. Part II: Choice of analytical methods," Applied spectroscopy, 62, 273-278 (2008).

4. Smith, Z. J., Strombom, S. and Wachsmann-Hogiu, S., "Multivariate optical computing using a digital micromirror device for fluorescence and Raman spectroscopy," Optics express, 19, 16950-16962, (2011).

5. Davis, B. M., Hemphill, A. J., Cebeci Maltas, D., Zipper, M. A.; Wang, P. and Ben-Amotz, D., "Multivariate hyperspectral Raman imaging using compressive detection," Analytical chemistry, 83, 5086-5092, (2011).

6. P. Berto, C. Scotté, F. Galland, H. Rigneault and H. B. de Aguiar. "Programmable single-pixel-based broadband stimulated Raman scattering," Opt. Letters, 9, 1696-1699, (2017).

7. Wilcox, D. S.; Buzzard, G. T.; Lucier, B. J.; Wang, P. and Ben-Amotz, D., "Photon level chemical classification using digital compressive detection," Analytica chimica acta, 755, 17-27 (2012).

8. Buzzard, G. T. and Lucier, B. J., Optimal filters for high-speed compressive detection in spectroscopy," IS\&T/SPIE Electronic Imaging, 8657, 865707-1-865707-10, (2013).

9. Wilcox, D. S.; Buzzard, G. T.; Lucier, B. J.; Rehrauer, O. G.; Wang, P. and Ben-Amotz, D., "Digital compressive chemical quantitation and hyperspectral imaging," Analyst, 138, 4982-4990, (2013).

10. Rehrauer, O. G.; Mankani, B. R.; Buzzard, G. T.; Lucier, B. J. and Ben-Amotz, D., "Fluorescence modeling for optimized-binary compressive detection Raman spectroscopy," Opt. Express, 23, 23935-23951, (2015).

11. Palkki, R. D. and Lanterman, A. D., "Chemical mixture estimation under a Poisson Raman spectroscopy model," Opt. Eng., 49, 113601113601-11, (2010).

12. Garthwaite,P.H. Jolliffe, I.T. and Jones, B. "Statistical Inference," (Printice Hall Europe, London, (1995). 